ARTICLE

https://doi.org/10.1038/s41467-019-13087-4

\title{
Hierarchical nanostructured aluminum alloy with ultrahigh strength and large plasticity
}

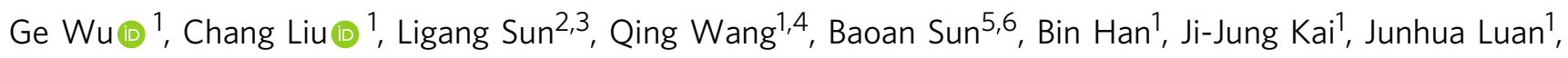

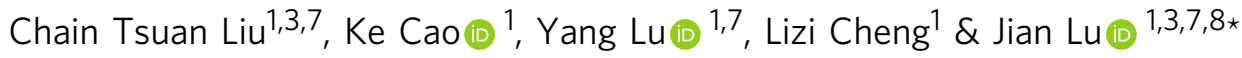

High strength and high ductility are often mutually exclusive properties for structural metallic materials. This is particularly important for aluminum (Al)-based alloys which are widely commercially employed. Here, we introduce a hierarchical nanostructured Al alloy with a structure of Al nanograins surrounded by nano-sized metallic glass (MG) shells. It achieves an ultrahigh yield strength of $1.2 \mathrm{GPa}$ in tension (1.7 GPa in compression) along with $15 \%$ plasticity in tension (over $70 \%$ in compression). The nano-sized MG phase facilitates such ultrahigh strength by impeding dislocation gliding from one nanograin to another, while continuous generation-movement-annihilation of dislocations in the Al nanograins and the flow behavior of the nano-sized MG phase result in increased plasticity. This plastic deformation mechanism is also an efficient way to decrease grain size to sub-10 $\mathrm{nm}$ size for low melting temperature metals like $\mathrm{Al}$, making this structural design one solution to the strength-plasticity trade-off.

\footnotetext{
${ }^{1}$ Department of Mechanical Engineering, City University of Hong Kong, Hong Kong, China. ${ }^{2}$ School of Science, Harbin Institute of Technology, Shenzhen 518055, China. ${ }^{3}$ Hong Kong Branch of National Precious Metals Material Engineering Research Centre, City University of Hong Kong, Hong Kong, China. ${ }^{4}$ Laboratory for Microstructures, Institute of Materials Science, Shanghai University, Shanghai 200072, China. ${ }^{5}$ Institute of Physics, Chinese Academy of Sciences, Beijing 100190, China. ${ }^{6}$ Songshan Lake Materials Laboratory, Dongguan, Guangdong 523808, China. ${ }^{7}$ Department of Materials Science and Engineering, City University of Hong Kong, Hong Kong, China. ${ }^{8}$ Centre for Advanced Structural Materials, Shenzhen Research Institute of City University of Hong Kong, Shenzhen 518057, China. *email: jianlu@cityu.edu.hk
} 
S tronger crystalline alloys are usually designed by controlling defects to hinder dislocation motion. These defects can be classified as point, line, interface, and volume defects ${ }^{1}$. Correspondingly, the mechanisms ${ }^{1,2}$ for making materials stronger are solid solution strengthening, dislocation strengthening, grain (or interphase) boundary strengthening, and precipitate (or dispersed reinforcement particle) strengthening. Strengthening approaches usually decrease ductility as a compromise $^{2}$. In order to overcome this strength-ductility trade-off, several strategies have been developed. For example, certain twin boundaries (TBs) allow dislocations to move in neighboring domains (twin or matrix) or glide along $\mathrm{TBs}^{3}$ and phase transformation induced plasticity (TRIP) effect may provide strain hardening ${ }^{4}$, thereby enhancing ductility. In contrast, noncrystalline solids do not possess slip systems and lattice dislocations due to the lack of long-range periodicity in their atomic structures $^{5}$. Thus, amorphous metallic glasses (MGs) have quite different deformation mechanisms when compared with their crystalline counterparts ${ }^{6}$. In absence of dislocation-mediated crystallographic slip, MGs manifest large elastic deformation of $2 \%$ prior to yielding and correspondingly exhibit high yield strength superior to crystalline alloys ${ }^{6}$. For example, the compressive strength of Co-based $\mathrm{MGs}^{7}$ can reach $\sim 5 \mathrm{GPa}$ and many Fe-based $\mathrm{MGs}^{8}$ show strength as large as $\sim 4 \mathrm{GPa}$. However, plastic deformation of MGs at ambient temperature is highly localized in shear bands 9 , thus leading to a catastrophic failure without any significant macroscopic ductility. Through heterogeneity nanostructuring ${ }^{10}$ or crystalline phase addition ${ }^{11}$ / transformation ${ }^{12,13}$, the ductility of MGs can be enhanced. The ductility can be increased to $7 \%$ after introducing a B2 phase $^{13}$ into the MG matrix. By amorphous/crystalline laminate nanostructuring, the plastic deformation can be increased to $10 \sim 30 \%{ }^{14-17}$. In these approaches, plasticity originates from the dislocation movement inside the crystalline phase and multiple shear bandings in the MG phase. However, the strength of these MG matrix composites (MGMC) have difficulty reaching that of their MG counterpart as a result of soft crystalline phases or the shear band softening effect, unless other strengthening mechanisms ${ }^{13}$ balance these softenings. If the size of the MGs is smaller than $100 \mathrm{~nm}$, the shear banding event can be fully suppressed $^{18,19}$, which contributes to an ideal strength ${ }^{20}$ and homogeneous plastic flow behavior of the MGs. Therefore, we hypothesize that with an extremely thin MG phase surrounding the crystalline phase, strain hardening of the crystalline phase and plastic flow of the nano-sized MG phase will contribute to both high strength and large ductility.

To realize such a material, we develop a hierarchical nanostructured $\mathrm{Al}$ alloy composed of face-centered-cubic $(f c c)$ nanograins surrounding by nano-sized MG shells. The grain boundaries are mostly replaced by the strong MG shells, which contributes to an ultrahigh strength of $1.7 \mathrm{GPa}$. During plastic deformation, the nano-sized MG phase exhibits flow behavior due to its small size. Dislocations are initiated from the nanograin/MG interfaces. Some dislocations pile up in the nanograins and provide strain hardening, while the majority of the dislocations move within the nanograins and annihilate at another nanograin/MG interface. This deformation mechanism contributes to the large plasticity.

\section{Results}

Structure design aided by molecular dynamic simulations. We conducted computer simulations to verify the hypothesis first, then a hierarchical nanostructure was built accordingly. $f c c$ metals are an ideal model since the dominant mode of deformation is dislocation glide and climb. The plastic deformation of aluminum
(Al) is based on this mechanism, and twinning is difficult to initiate due to its high stacking fault energy ${ }^{21}$. The universal $f c c$ structure and the dislocation-slip-based plastic deformation mechanism led to our material design of hierarchical nanostructured $\mathrm{Al}$ alloy. The hierarchical nanostructure of the $\mathrm{Al}$ nanograins embedded in the $\mathrm{Al}_{85} \mathrm{Ni}_{15} \mathrm{MG}$ was designed and then simulated by the molecular dynamic (MD) (Fig. 1a and Supplementary Fig. 1). A simulated compression test was performed on the structure. Our results show that $\mathrm{Al}$ nanograins become shorter and wider at a strain of 30\% (Fig. 1b), without any global shear of the whole structure or cavitation generation in the local area. This uniform plastic deformation is rarely seen in nanocrystalline, amorphous ${ }^{5}$ or glass-crystal nanocomposite ${ }^{22}$ materials, which usually possess high strength yet limited plasticity. Dislocations are initiated from the glass/crystal interface, indicated by the locally largest atomic shear strain on the interface (Fig. 1c, highlighted by the orange arrow). The large atomic shear strain in the amorphous phase indicates plastic flow behavior. Meanwhile, dislocation slip dominates the plastic deformation mechanism of the $\mathrm{Al}$ nanograins, leaving local larger atomic shear strain trajectories (Fig. 1c, highlighted by light blue arrow). The cross slip of dislocations offset the atomic shear strain, resulting in a serious underestimation of the number of dislocations during deformation. This effect is shown in a structure evolution movie (Supplementary Movie 1) of 'G1' in Fig. 1b. Despite previous studies reporting that glass-crystal nano-dual-phase nanostructuring could provide ultrahigh strength yet lose plasticity ${ }^{22}$, that study shows the limited plasticity may be attributed to the brittle nature of intermetallic nanocrystals. By substituting the brittle intermetallic phase with a ductile $f c c$ Al phase, dislocation motion in the $\mathrm{Al}$ phase could initiate plastic deformation in the hierarchical nanostructured $\mathrm{Al}$ alloy. In addition, we fabricated another hierarchical nanostructure model with much lower fraction of MG phase ( 1/10 size of the crystalline phase) and which shows similar deformation behavior (Supplementary Fig. 2).

Microstructure characterization. The 3D hierarchical structure designed by MD simulation has shown large plasticity, and the $\mathrm{Al}$ alloy exhibiting such characteristic can be successfully fabricated. In former reports, the $\mathrm{Al}$ content is usually $80-90 \%$ (at.\%) in conventional Al-based $\mathrm{MGs}^{23}$. If the $\mathrm{Al}$ content exceeds $90 \%$, nearly pure $f c c \mathrm{Al}$ crystals could be generated in the MG matrix ${ }^{24}$. Therefore, we increased the $\mathrm{Al}$ content to $95 \%$ (at.\%) in our $\mathrm{Al}$ $\mathrm{Ni}-\mathrm{Y}$ alloy to obtain a glass-crystal dual-phase. The average composition of the hierarchical nanostructured $\mathrm{Al}$ alloy is $\mathrm{Al}_{95} \mathrm{Ni}_{2} \mathrm{Y}_{3}$ (at.\%). The $f c c \mathrm{Al}$ nanograins with a diameter of $\sim 40$ $\mathrm{nm}$ are dispersed uniformly across the whole specimen. The size of the nanograins was discerned from the darkest contrast regions in Fig. 1e. The structural unit of the hierarchical nanostructured $\mathrm{Al}$ alloy is tabular-like with $\sim 40 \mathrm{~nm}$ width and $\sim 100 \mathrm{~nm}$ length. Therefore, there are 2-3 nanograins in each structural unit. The interfaces between the structural units (gray lines in Fig. 1e) are not grain boundaries (GBs) as in conventional polycrystalline materials, but are a secondary amorphous phase with a thickness of $\sim 4 \mathrm{~nm}$ (Fig. 1h). The maze-like pattern in the high resolution transmission electron microscope (HRTEM) image and diffuse pattern in the corresponding FFT image indicate the amorphous structure. Furthermore, to unambiguously reveal the structure difference between the amorphous nanolayer and the conventional GB, we annealed the hierarchical nanostructured $\mathrm{Al}$ alloy at $300^{\circ} \mathrm{C}$ for $2 \mathrm{~h}$ to fully crystallize the amorphous phase. The HRTEM image of the grain-grain interface is shown in Supplementary Fig. 3, which demonstrates a distinct difference from the amorphous nanolayer. The amorphous phase in the as-deposited 
a Simulation
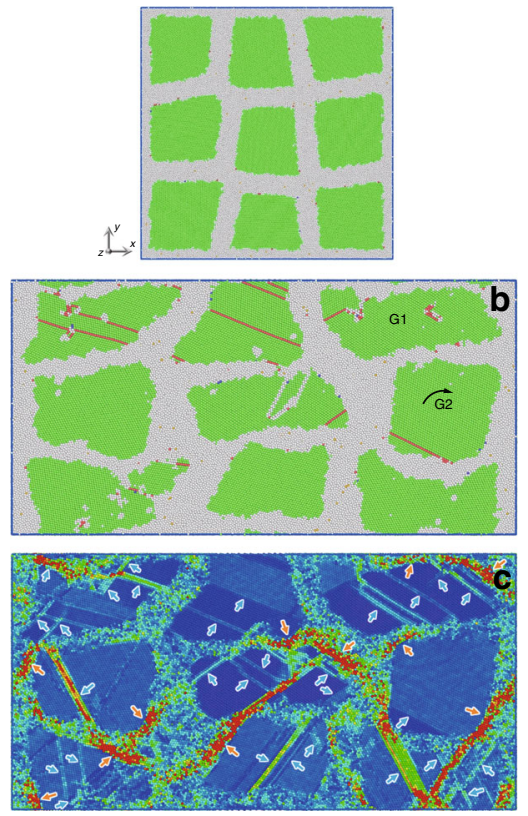

d As-deposited sample

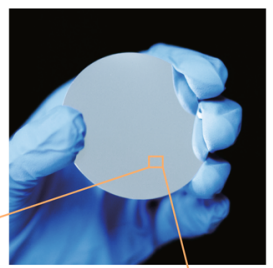

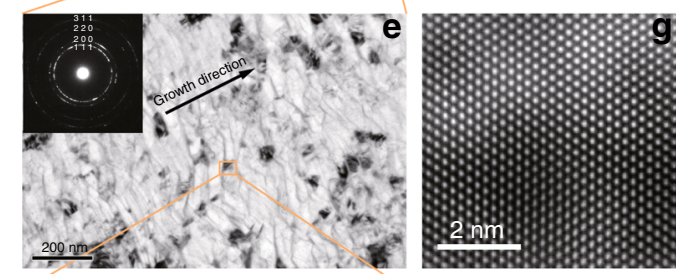
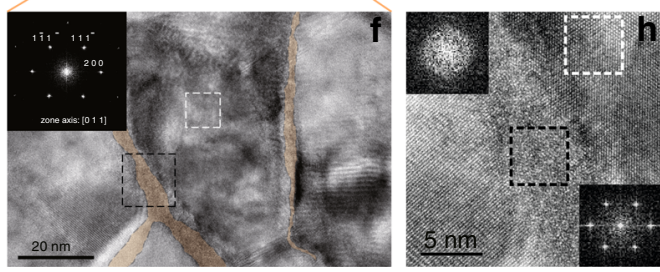

Fig. 1 Hierarchical nanostructure guided from computer simulation. a The atomistic model of the glass-crystal hierarchical nanostructure composed of amorphous $\mathrm{Al}_{85} \mathrm{Ni}_{15}$ and $\mathrm{Al}$ nanograins. $\mathbf{b} \mathrm{MD}$ simulated atomic snapshot and $\mathbf{c}$ the corresponding atomic shear strain distribution of the hierarchical nanostructured $\mathrm{Al}$ alloy at a strain of 30\%. 'G1' and ' $\mathrm{G} 2$ ' in $\mathbf{b}$ represent a flattened 'grain 1' and a rotated 'grain 2', respectively. The typical dislocation slips in 'G1' contribute to plastic deformation. Large rotation with little dislocation propagation in 'G2' may also accommodate the plastic deformation. $\mathbf{d}$ Optical image of the hierarchical nanostructured Al alloy. e Cross-sectional TEM image of the hierarchical nanostructured Al alloy, showing Al nanograins surrounded with light-dark contrasted grain-grain interfaces. The selected area electron diffraction (SAED) pattern in the inset denotes the polycrystalline structure. f HRTEM image of the hierarchical nanostructured Al alloy, showing a crystalline Al nanograin is surrounded by amorphous phase (post-colored by light yellow). The inset is fast Fourier transformation (FFT) image of the white dashed rectangle region in the main image, showing the fcc structure with zone axis of [0 11 1]. $\mathbf{g}, \mathbf{h}$ are the zoomed-in images of white and black dashed rectangle regions in (f), respectively. $\mathbf{g}$ Shows a defect-free fcc structure. $\mathbf{h}$ Shows a nano-sized amorphous phase forms between the two grains. The FFT image (lower right inset of $\mathbf{h}$ ) of the crystalline region (white dashed rectangle) reveals pronounced spots pattern. By contrast, the FFT image (upper left inset of $\mathbf{h}$ ) of the black dashed rectangle region in $\mathbf{h}$ shows a diffuse pattern, indicating the amorphous structure. The termination ripples feature may be due to the small sampling size

a

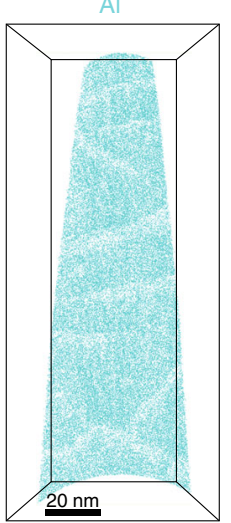

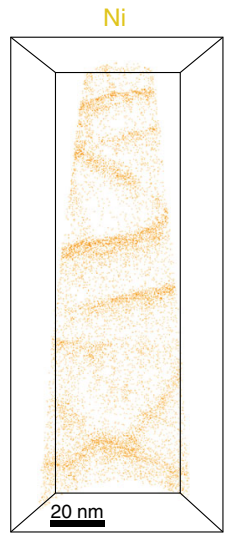

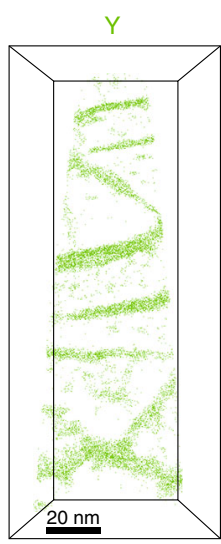

b

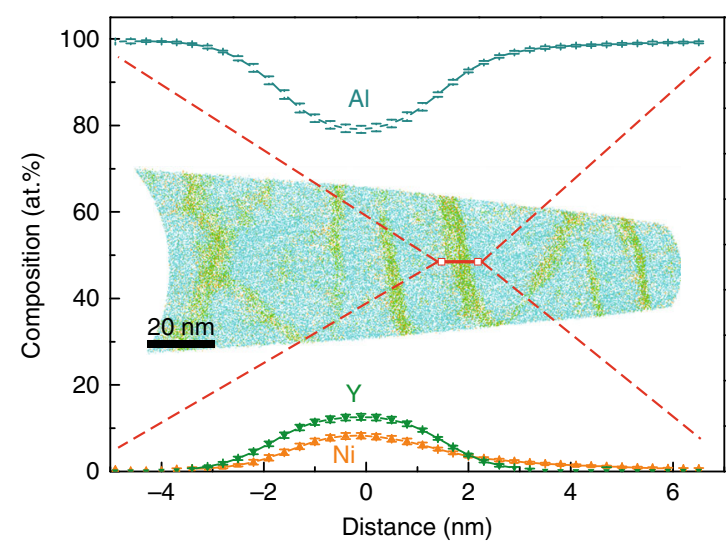

Fig. 2 3D heterogeneity of the hierarchical nanostructured Al alloy. a Thin slice from three-dimensional reconstruction of an APT dataset, showing Ni and Y elements enrichment in the interface between pure Al nanograins. b Atom distribution showing the composition change across the selected interface. The error bars are standard deviations

sample possesses a thickness of $\sim 4 \mathrm{~nm}$ and length of tens to hundred nanometers, which is larger than the $\sim 1 \mathrm{~nm}$-sized short/ medium range ordered clusters in $\mathrm{MGs}^{25,26}$. It is similar to an amorphous intergranular film, a type of GB complexion ${ }^{27}$, which is usually $1-5 \mathrm{~nm}$ thick ${ }^{28,29}$. Because the composition of this amorphous phase (Fig. 2) is a good glass former, it manifests as an MG phase.
Element distributions in the MG phase. The hierarchical nanostructured Al alloy exhibits chemical composition heterogeneity (Fig. 2). The composition of the amorphous phase is $\mathrm{Al}_{80} \mathrm{Ni}_{8} \mathrm{Y}_{12}$ (at.\%), and the nanograins are pure $\mathrm{Al}$, which was investigated by atomic probe tomography (APT). The volume fraction of the amorphous phase is calculated to be $25 \%$ based on the compositions of the whole material $\left(\mathrm{Al}_{95} \mathrm{Ni}_{2} \mathrm{Y}_{3}\right)$, the 
crystalline phase $\left(\mathrm{Al}_{100} \mathrm{Ni}_{0} \mathrm{Y}_{0}\right)$ and the MG phase $\left(\mathrm{Al}_{80} \mathrm{Ni}_{8} \mathrm{Y}_{12}\right)$. Furthermore, it is easy to calculate the volume fraction from the reconstruction of the APT results, which is estimated to be $20 \%$. While $\mathrm{Al}_{80} \mathrm{Ni}_{8} \mathrm{Y}_{12}$ is a typical glass forming composition ${ }^{23}$, the amorphous $\mathrm{Al}_{80} \mathrm{Ni}_{8} \mathrm{Y}_{12}$ phase is formed under fast cooling condition during the fabrication process, leaving $\mathrm{Al}$ nanocrystals with poor glass forming ability. The alloy system is not fully amorphous because the $\mathrm{Al}$ content is $95 \%$, which is beyond the glass forming region for Al-based MGs (Al content of $80-90 \% 27$ ). The formation of Al nanocrystals leads to solute rejection into the MG phase, which in turn stabilizes it. Al nanocrystals do not grow into columns as other alloy films $\mathrm{do}^{30}$ in the sputtering process, which could be attributed to the fact that the nano-sized MG phase acts as an interruption barrier, disrupting the preferred growth pattern of Al nanocrystals. It should be noted that the $f c c$ Al phase in the Al-based MGMCs can be generated by composition control ${ }^{24}$ or post heat treatment of $\mathrm{MGs}^{31}$. However, the hierarchical nanostructure with such extremely thin MG phase reported here, which is essential for good mechanical properties, has rarely been seen in previous reports ${ }^{24,31}$.

Mechanical properties. The overall high strength and flexibility properties of the hierarchical nanostructured $\mathrm{Al}$ alloy are illustrated in Supplementary Movie 2. The alloy's mechanical properties were analyzed by scanning electron microscope (SEM) in situ compression and tension tests under identical conditions (sample size and loading rate) for the hierarchical nanostructured $\mathrm{Al}$ alloy, Al-based MG and nanocrystalline Al. Compressive engineering stress-strain curves (Fig. 3) show that nanocrystalline $\mathrm{Al}$ has a yield strength of only $0.2 \mathrm{GPa}$ with discontinuous plastic flow while Al-based MG has a yield strength of $1.0 \mathrm{GPa}$ without any plasticity. The mechanical performance of both nanocrystalline $\mathrm{Al}$ and $\mathrm{Al}$-based $\mathrm{MG}$ agrees well with previous reports ${ }^{23,32}$. In contrast, the hierarchical nanostructured $\mathrm{Al}$ alloy displays an ultrahigh yield strength of $1.7 \mathrm{GPa}$, and a homogeneous large plastic deformation with a strain of over $70 \%$. Larger samples of the hierarchical nanostructured $\mathrm{Al}$ alloy $(8 \mu \mathrm{m}$ diameter) were tested to eliminate the possibility of a size effect (Supplementary Fig. 4). The sample size effect on mechanical properties usually appears when the size of the sample is comparable to its defect. There is a low probability of defects existing inside the small sample $^{33}$, which induces a near-ideal strength ${ }^{20,34}$. It is believed

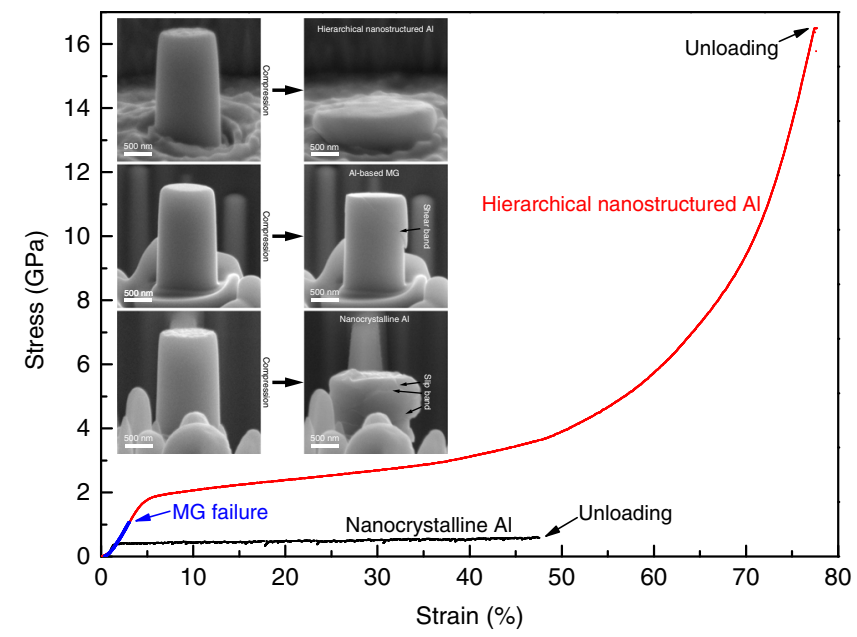

Fig. 3 Mechanical property of the hierarchical nanostructured Al alloy. Compressive engineering stress-strain curves of the hierarchical nanostructured Al alloy, Al-based MG and nanocrystalline Al pillar samples with the same diameter of $1 \mu \mathrm{m}$. The insets are the SEM images of the samples before and after compression that the $3 \mu \mathrm{m}$-diameter sample is able to exclude the size effect for a nanotwinned $\mathrm{Al}$ alloys ${ }^{30}$. Because the size of our $8 \mu \mathrm{m}$-diameter sample is more than 80 times larger than that of its structure unit, it may possess little sample size effect. After compression, nanocrystalline $\mathrm{Al}$ and $\mathrm{Al}$-based $\mathrm{MG}$ reveal slip bands and shear bands, respectively, which are typical signatures of inhomogeneous deformation. However, the hierarchical nanostructured $\mathrm{Al}$ shows homogeneous deformation, which is confirmed by both SEM images of the pillar sample after compression and the SEM in situ compression movie (Supplementary Movie 3). Furthermore, in the SEM in situ tension test (Supplementary Fig. 5 and Movie 4), strength and ductility in tension are revealed. True stress and true strain are calculated by measuring the instantaneous load and gauge width ${ }^{18}$. The hierarchical nanostructured Al alloy shows a tensile yield strength of $1.2 \mathrm{GPa}$ with $17 \%$ fracture strain (60\% ultimate true strain). While nanocrystalline materials have relative higher strength than their coarse-grained counterparts, shear localization always takes place ${ }^{32}$. MGs have a much higher strength than their crystalline counterparts, yet inevitably display limited plasticity as a result of shear band instability ${ }^{6}$. By combining these two nanostructural units to form the hierarchical nanostructure, we can achieve ultrahigh strength with homogeneous deformation. The GBs usually act as dislocation sources for the nanocrystalline alloys ${ }^{35,36}$. In the hierarchical nanostructured $\mathrm{Al}$ alloy, however, the GBs are mostly replaced by the nano-sized MG phase. The extremely small-sized MG is believed to reach near-ideal strength ${ }^{20}$, which effectively impede the dislocation generation in the crystalline phase, thus enhancing the yield strength. The detailed plastic deformation mechanisms will be discussed further below. It is worthwhile to note that the focused ion beam (FIB) current was controlled to be as small as possible (1.5 pA at the final milling stage) to minimize the surface damage of the micro-pillar samples by ion beam. It is realized that the surface damage layer generated at $30 \mathrm{kV}$ Ga FIB milling is $6 \mathrm{~nm}$ thick ${ }^{37}$ for $\mathrm{Al}$ alloys, which is thin compared to the diameter of the micro-pillar. Although it is reported that the surface damage effect may lead to yield strength decrease (less than $20 \%$ for $1 \mu \mathrm{m}$-sized pillar) of nanocrystalline $\mathrm{Al}$, it does not influence plasticity ${ }^{38}$. In order to exclude the surface damage effect on the mechanical property of the glass-crystal hierarchical nanostructure in the specific alloy system, the same compression test was conducted on a hierarchical nanostructured $\mathrm{Mg}$ alloy micro-pillar (Supplementary Fig. 6). It also shows much higher strength and plasticity than that of its MG counterpart. Therefore, the hierarchical nanostructure overcomes strength-plasticity trade-off, which is a long-standing goal in structural materials research ${ }^{39,40}$. Furthermore, Al is an important light-weight structural material with extensive applications in different industries. By comparing specific yield strength vs. $E / \sigma_{y}$ of the hierarchical nanostructured Al alloy with other ultrastrong materials (Supplementary Fig. 7), our study shows the achievement of ultrahigh specific strength by the hierarchical nanostructure.

\section{Discussion}

From the compressive true stress-strain curve (Supplementary Fig. 8a), strain hardening exists from 70 to $150 \%$ true strain. Such strain hardening does not come from the lateral constraint effect due to the friction between indenter and the top surface of the pillar, which is usually seen in later stage compression ${ }^{41}$. To verify this, the strength of a pre-compressed larger pillar was tested. A larger pillar with a diameter of $2 \mu \mathrm{m}$ and a height of $4 \mu \mathrm{m}$ was compressed to about half of its original height, then was milled using FIB to have a $2: 1$ aspect-ratio, that is $1 \mu \mathrm{m}$-diameter pillar (illustrated in Supplementary Fig. $8 \mathrm{~b}$ inset images). The 

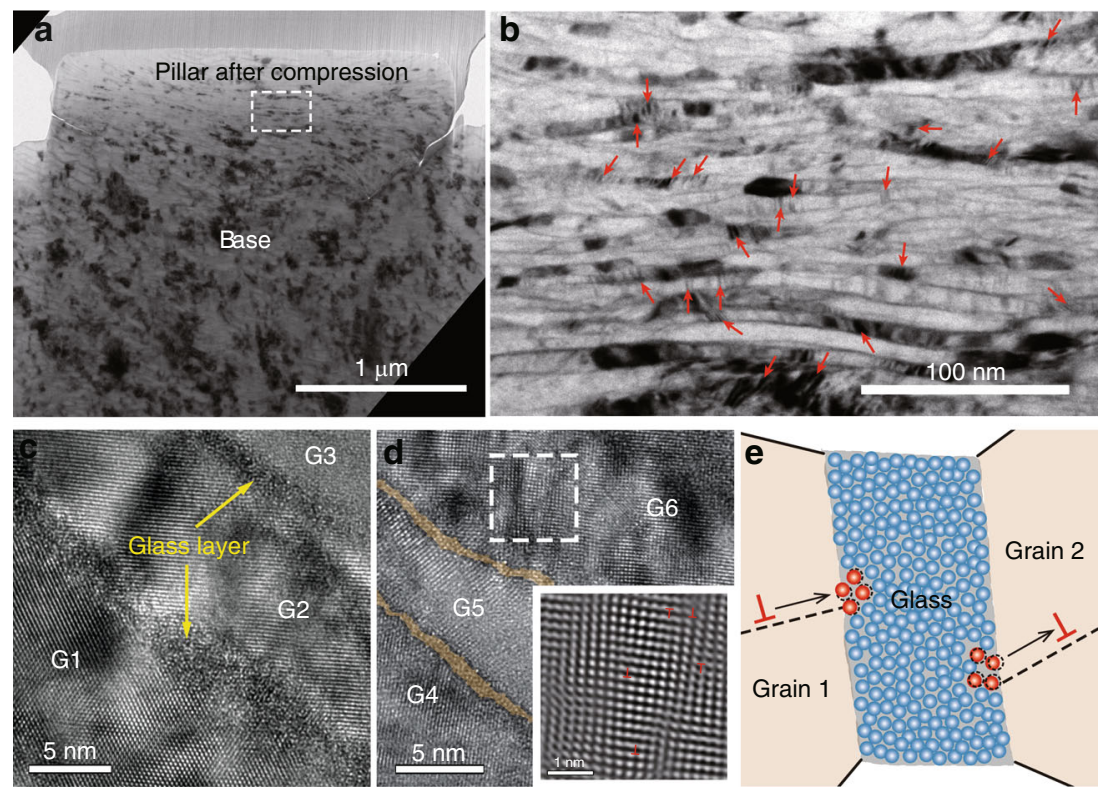

Fig. 4 Plastic deformation mechanism of the hierarchical nanostructured Al alloy. a Cross-sectional TEM image of a $1 \mu \mathrm{m}$-diameter pillar after compression. b Enlarged TEM image from white dashed rectangle area in (a). The red arrows indicate the positions of some dark regions. c HRTEM image near nanolamellar grains $\mathrm{G} 1, \mathrm{G} 2$, and $\mathrm{G} 3$, clearly demonstrates the existence of the amorphous phase (glass layer) after deformation. $\mathbf{d}$ HRTEM image near nanolamellar grains G4, G5, and G6. The nano-sized MG phase is colored by light yellow. The lower right inset is inverse Fourier transformation (IFT) image of the dashed square area in the main image, showing some of the dislocations ' $\perp$ '. e Illustration of dislocations' activities interacted with the nano-sized MG phase. A dislocation (' $\perp$ ') is generated on the glass-grain 2 interface and then moves inside grain 2. Another dislocation (' $\perp$ ') moves inside grain 1 and then is absorbed by the atoms on the edge of the nano-sized MG phase (dislocation annihilation). The red and blue spheres represent mobile and less mobile atoms respectively. The dashed circles represent the original positions of the mobile atoms. The black arrows denote the motion directions of the dislocations

compression experiment was conducted on this pillar again. The result shows a higher strength when compared with the same dimension pillar milled from the as-received sample, thus confirming the existence of strain hardening (Supplementary Fig. 8b).

In tension tests, strain hardening appears from 10 to $55 \%$ true strain (Supplementary Fig. 5 and Movie 4), and appears in both the compression and the tension for the hierarchical nanostructured $\mathrm{Al}$ alloy. It is generally known that strain hardening has difficulty taking place in amorphous and nanocrystalline materials. The reason is that in amorphous materials, work softening due to the shear-dilation prevails during plastic deformation ${ }^{6}$, and in nanocrystalline materials, plastic deformation is usually associated with grain boundary (GB) activity ${ }^{42,43}$, such as grain growth, GB sliding or grain rotation that lead to strain softening. Therefore, the deformation mechanism of the hierarchical nanostructured Al alloy should be different from that of monolithic amorphous or nanocrystalline phases. To understand the deformation mechanism, the microstructure of the compressed pillar with a true strain of $150 \%$ (engineering strain of $76 \%$ ) was investigated by TEM (Fig. 4). The overall compressed pillar shows a flat-barrel shape without any shear plane/offset (Fig. 4a), which indicates homogeneous plastic deformation. In the larger magnification TEM image of the compressed pillar (Fig. 4b), the nanograins show the lamellar structure, and the vertical width of the nanograins decreases from $\sim 40$ to $\sim 8 \mathrm{~nm}$ after compression. Nano-lamellar grains orientated with crystallographically lowindex planes (Fig. 4c) is helpful to reveal the extremely thin MG layer between them. It shows that the width of the nano-sized MG phase decrease from $\sim 4$ to $\sim 1 \mathrm{~nm}$ after plastic deformation. This confirms the existence of fully homogeneous plastic flow of the nano-sized MG phase without any shear band ${ }^{18}$.

Previous investigations pointed out that due to thermally activated GB migration, grain refinement of low melting temperature metals by straining is extremely difficult ${ }^{44}$. The smallest grain size of $65 \mathrm{~nm}$ for $\mathrm{Al}$ was achieved by surface mechanical grinding treatment ${ }^{44}$. In our study, $\sim 8 \mathrm{~nm}$ grain size was achieved in a compressed hierarchical nanostructured $\mathrm{Al}$. In other words, the nano-sized MG phase in the hierarchical nanostructured $\mathrm{Al}$ effectively impedes $\mathrm{GB}$ migration, which prevents softening mechanisms (such as grain growth) from taking place. It is known that the softening of the conventional nanocrystalline materials originates from the instability of the $\mathrm{GBs}^{45}$. However, for the hierarchical nanostructured $\mathrm{Al}$ alloy, most of the GBs are replaced by a nano-sized MG phase, which impedes dislocations motion from one grain to another, contributing to the alloy's ultrahigh strength. In the deformed sample, plenty of striped dark regions can be seen from the bright field (BF)-TEM image (Fig. 4b). These dark regions result from bending of the crystals to orientations closer to a good two-beam condition. In the HRTEM image (Fig. 4d), it is clear that the dark regions contain high-density dislocations, which are regarded as dislocations piled up inside nanograins. This mechanism contributes to the strain hardening of the nanograins. Furthermore, the homogeneous plastic flow in the nano-sized MG phase is notable, because bulk MGs usually exhibit limited plasticity due to shearband instability. This phenomenon may be attributed to MGs' intrinsic plastic deformation ability in extremely small size ${ }^{18,19}$. The flow stress of the nano-sized MG phase may reach to theoretical strength during plastic flow ${ }^{18}$. This indicates that the nano-sized MG phase is still strong during plastic deformation and is able to prevent the softening mechanism of the nanograins, such as GB migration in the conventional nanocrystalline materials. Moreover, its homogeneous flow behavior accommodates the large plastic deformation of the nanograins, which prevents shear deformation mechanism, such as stress localization. The interface usually plays a key role for crack deflection, thus 
enhancing toughness ${ }^{46}$. In this hierarchical nanostructured $\mathrm{Al}$ alloy, the MG phase on the interface has plastic deformation and no void or crack is seen in the highly deformed sample (Fig. 4), which may indicate a high toughness of the alloy.

To further demonstrate the dynamic structure evolution during compression, a TEM in situ compression experiment was performed on a nanopillar (Supplementary Movie 5). During plastic deformation, dislocations can be generated on the glass/crystal interfaces (dark contrasts denoted by yellow solid circles in Supplementary Movie 5) and move inside the grains. Some of them may pile up inside the grains, and some may move towards the nano-sized MG phase. Because the nano-sized MG phase can flow at such high stress level ${ }^{18,19}$, the mobile atoms on the edge of the MG phase are able to act as the ideal sink for dislocations when they are encountered. As a consequence, these dislocations disappear at the interfaces and the grain regions near the glass/ crystal interfaces return to quasi-dislocation free (denoted by yellow dashed circles in Supplementary Movie 5). Since these kinds of dislocations temporarily appear during plastic deformation, they are termed transitory-dislocations. The continuous generation-movement-annihilation of transitory-dislocations (illustrated in Fig. 4e) contributes to the homogenous deformation of the hierarchical nanostructured $\mathrm{Al}$ alloy, and this mechanism is further revealed in an MD simulation (Supplementary Figs. 9-13). Similar dislocation movement in the nanograins also appears in a TEM in situ tension test (Supplementary Movie 6). The real-time Supplementary Movie 6 was captured after a tension pulse with a speed of $500 \mathrm{~nm}$ per second.

In summary, a hierarchical nanostructured $\mathrm{Al}$ alloy was developed with the assistance of computer simulation. The structure unit is a pair of $\sim 40 \mathrm{~nm}$-sized nanograins surrounded by $\sim 4 \mathrm{~nm}$-thick MG phase. The alloy achieves ultrahigh yield strength and large plasticity in both tension and compression. Its ultrahigh strength is a result of the nano-sized MG phase impeding dislocation gliding from one nanograin to another nanograin in the neighboring structure unit, while its large plasticity is a result of the continuous transitory-dislocations' generation-movement-annihilation in the nanograins and the intrinsic plastic flow of the nano-sized MG phase. Our results illustrate a hierarchical nanostructure approach in material engineering and may contribute to not only the development of tough lightweight alloys but also the applications of microelectromechanical systems (MEMS) flexible wearable devices.

\section{Methods}

Fabrication of the materials. We use magnetron sputtering as the fabrication method. We use an $\mathrm{Al}_{92} \mathrm{Ni}_{2} \mathrm{Y}_{6}$ (at.\%) alloy target with the purity of $99.9 \%$. Then the hierarchical nanostructured $\mathrm{Al}_{95} \mathrm{Ni}_{2} \mathrm{Y}_{3}$ (at.\%) with the thickness of 17-50 $\mu \mathrm{m}$ were deposited on the $\mathrm{Si}\left(\begin{array}{lll}0 & 0 & 1\end{array}\right)$ substrate. In the sputtering process, the Ar pressure was $0.2 \mathrm{~Pa}$; the deposition rate was $12.5 \mathrm{~nm}$ per minute; the substrate bias voltage was $-50 \mathrm{~V}$. The $\mathrm{Al}_{87} \mathrm{Ni}_{5} \mathrm{Y}_{8}$ (at.\%) MG was fabricated by melt-spun method. A pure $\mathrm{Al}$ plate with thickness of $1.5 \mathrm{~mm}$ was treated by surface mechanical attrition treatment to generate nanocrystalline grains on the surface. The nanocrystalline layer with uniform equiaxed grain size of $\sim 300 \mathrm{~nm}$ has a thickness of $\sim 50 \mu \mathrm{m}$. The $4 \mu \mathrm{m}$ thick Mg-based $\mathrm{MG}\left(\mathrm{Mg}_{58} \mathrm{Zn}_{37} \mathrm{Ca}_{5}\right.$ (at.\%)) film was fabricated by magnetron sputtering using a $\mathrm{Mg}_{60} \mathrm{Zn}_{35} \mathrm{Ca}_{5}$ (at.\%) alloy target with $99.9 \%$ purity. During the sputtering process, the Ar pressure was $0.2 \mathrm{~Pa}$; the deposition rate was $10.6 \mathrm{~nm}$ per minute; the substrate bias voltage was $-50 \mathrm{~V}$. The Mg-based MG was then annealed at $300^{\circ} \mathrm{C}$ to generate a glass-crystal hierarchical nanostructure. The thickness of the materials is large enough to fabricate micro-pillar samples by using focused ion beam (FIB).

Structural characterization. The structure of the hierarchical nanostructured Al alloy was studied by TEM. We used a JEM 2100F FEG transmission electron microscope (from JEOL), operated at $200 \mathrm{kV}$, for TEM analysis. The thin-foil TEM samples were prepared with a FEI Scios ${ }^{\text {Tx }}$ DualBeam $^{\text {Ts }}$ FIB, the final milling voltage/ current was $2 \mathrm{kV} / 34 \mathrm{pA}$, which was small enough to avoid potential crystallization or amorphization. Needle-shaped specimens required for APT were fabricated by lift-outs and annular milled by FIB. The APT characterizations were performed in a local electrode atom probe (CAMEACA LEAP 5000XR). The specimens were analyzed at $50 \mathrm{~K}$ in voltage mode, a pulse repetition rate of $200 \mathrm{kHz}$, a pulse fraction of $20 \%$, and an evaporation detection rate of $0.4 \%$ atom per pulse. Imago Visualization and Analysis Software (IVAS) version 3.8 was used for creating the $3 \mathrm{D}$ reconstructions and data analysis.

Mechanical characterization. Nanoindentation was performed by Hysitron TI950 nanoindenter with a Berkovich tip. The indentation depth was kept below $10 \%$ of the film thickness to avoid the substrate effect. Micro-pillar and micro-dog-bone shaped samples were fabricated by FIB, with $30 \mathrm{kV} / 1.5 \mathrm{pA}$ as the final milling condition. The height of the pillars was maintained smaller than the layer thickness. The aspect ratio (height/diameter) of the pillar was 2, and the taper angle of each nanopillar was less than $1.5^{\circ}$. Tension samples have square column crosssections with gauge width of $2 \mu \mathrm{m}$, aspect ratio of 2 . We conducted SEM in situ compression/tension tests at room temperature using a PI 85 PicoIndenter (Hysitron Inc.) with diamond punch/gripper inside a FEI Quanta 450 FEG scanning electron microscope, under displacement-control mode and at a strain rate of $1 \times 10^{-3}-5 \times 10^{-3} \mathrm{~s}^{-1}$. During deformation before yielding in SEM in situ tension, the true stress and true strain were converted by using the equations: ${ }^{18} \sigma_{\mathrm{T}}=$ $\sigma_{\mathrm{E}}\left(1+\varepsilon_{\mathrm{E}}\right)$ and $\varepsilon_{\mathrm{T}}=\ln \left(1+\varepsilon_{\mathrm{E}}\right)$, where $\sigma_{\mathrm{T}}, \sigma_{\mathrm{E}}, \varepsilon_{\mathrm{E}}$, and $\varepsilon_{\mathrm{T}}$ are the true stress, engineering stress, engineering strain, and true strain, respectively. After yielding, the instantaneous gauge width was measured from the in situ movie, and the true stress and true strain were calculated by using the equations: ${ }^{1} \sigma_{\mathrm{T}}=F / A_{\mathrm{i}}$ and $\varepsilon_{\mathrm{T}}=\ln \left(A_{0} / A_{\mathrm{i}}\right)$ where $A_{0}$ and $A_{\mathrm{i}}$ are the gauge cross-sectional areas initial and during plastic deformation, respectively. TEM in situ uniaxial tension tests was conducted at room temperature using a Gatan 654 straining holder inside a JEM 2100F FEG transmission electron microscope (from JEOL), operated at $200 \mathrm{kV}$. TEM in situ compression tests was conducted at room temperature using PI 95 PicoIndenter (Hysitron Inc.) with $1 \mu \mathrm{m}$-diameter diamond punch inside the TEM, operated at $200 \mathrm{kV}$. The displacement rate was $2 \mathrm{~nm}$ per second.

MD simulations. MD simulations were performed using LAMMPS package ${ }^{47}$ to study the binary Al-Ni system. The EAM potential developed by Pun et al. ${ }^{48}$ was employed to describe the atomic interactions within the Al-Ni system. With the consideration of the time scale limitation of MD simulation for nanocrystallization compared with the realistic formation process of nanometer-sized glass-crystal hierarchical nanostructure, we tried to construct the hierarchical nanostructured Al-based samples with the following process: a small cubic $\mathrm{Al}_{85} \mathrm{Ni}_{15}$ sample of (13,500 atoms) with periodic boundary conditions (PBCs) along all three dimensions is firstly equilibrated at $1800 \mathrm{~K}$ for $5 \mathrm{~ns}$ and then quenched to $300 \mathrm{~K}$ at a cooling rate of $10^{11} \mathrm{~K} / \mathrm{s}$, at 0 bar external pressure and under a Nosé-Hoover thermostat. Finally, the amorphous sample is relaxed at $300 \mathrm{~K}$ for $2 \mathrm{~ns}$. We analyzed the equilibrated configuration of $\mathrm{Al}_{85} \mathrm{Ni}_{15}$. Supplementary Fig. 1a shows the variation of volume of $\mathrm{Al}_{85} \mathrm{Ni}_{15}$ from $1800 \mathrm{~K}$ to $300 \mathrm{~K}$. The liquid to solid/glassy state transition point can clearly be observed by detecting the variation of volume during quenching. As observed from Supplementary Fig. 1b, the partial RDF curves of $\mathrm{Al}_{85} \mathrm{Ni}_{15}$ exhibits the main peaks of $\mathrm{Al}-\mathrm{Al}, \mathrm{Al}-\mathrm{Ni}$, and $\mathrm{Ni}-\mathrm{Ni}$ at $\sim 2.76 \AA$, $\sim 2.55 \AA$ and $\sim 2.61 \AA$. These structure information is consistent with previous ab initio MD results ${ }^{49,50}$. Thus, the final relaxed configuration of $\mathrm{Al}_{85} \mathrm{Ni}_{15}$ could be a typical $\mathrm{Al}-$ based MG. For the mechanical test, a large sample is constructed by replications of the 13,500 atom configurations $\left(\sim 39.0(\mathrm{x}) \times 39.0(\mathrm{y}) \times 14.3(\mathrm{z}) \mathrm{nm}^{3}\right)$. Then the single crystalline pure-Al columnar grains with grain size about $10 \mathrm{~nm}$ is modeled and embedded into the quenched $\mathrm{Al}_{85} \mathrm{Ni}_{15}$ sample with a Voronoi diagram, controlling the thickness of $\mathrm{Al}_{85} \mathrm{Ni}_{15}$ about $3 \mathrm{~nm}$. The sample contains about 1,320,000 atoms. This combined sample is further annealed for $0.5 \mathrm{~ns}$ before the tensile test. This annealing treatment is supposed to eliminate potential artifacts in the hierarchical nanostructured sample (Supplementary Fig. 1c). To compare the deformation mechanism between the hierarchical nanostructured Al-based alloy and the polycrystalline $\mathrm{Al}$, a polycrystalline sample of $\mathrm{Al}$ is also built, with the same grain size, shapes, distributions, and lattice orientations as the hierarchical nanostructured one (Supplementary Fig. 1d). For compression loading, PBCs are imposed along three directions. A constant strain rate of $\sim 2.5 \times 10^{8} \mathrm{~s}^{-1}$ along $x$ - or $y$-direction is imposed at a temperature of $300 \mathrm{~K}$.

To monitor plastic shearing during mechanical deformation, the local Von Mises atomic shear strain ${ }^{51}$ in the samples is calculated. To carry out geometrical analysis on the atomic configurations, we have adopted various methods such as Voronoi tessellation method for local motif analysis, radial distribution function (RDF) for identification of amorphous phase, atomic coordination numbers (CNs) and common neighbor analysis (CNA $)^{52}$, especially for crystal structure characterization.

Another model with crystalline phase size of $\sim 25 \mathrm{~nm}$ and MG thickness of $\sim 2.5$ nm was designed, and uniaxial compression was simulated to evaluate the possible size effect on the plastic deformation of this Al-Ni dual-phase structure (Supplementary Fig. 2). In this simulated sample, the thickness of the MG phase approaches $\sim 1 / 10^{\text {th }}$ of the crystalline phase, which is comparable to that of our experimental samples. Although the strain rates $\left(\sim 1.0 \times 10^{8} \mathrm{~s}^{-1}\right)$ in MD simulation are higher than that in experiments, it is generally accepted that simulation with the strain rate of this order is able to clarify the deformation mechanisms of the materials ${ }^{53,54}$. The atom configurations of the Al-Ni dual-phase sample before and after deformation are shown in Supplementary Fig. 2. The common neighbor analysis (CNA) and atomic shear strain coloring methods are employed. The 
dislocation-slip-based plastic deformation mode is clearly observed, similar to the results for a simulated sample with much larger fraction of MG phase (Fig. 1a-c). Furthermore, the vertical width of the grains decreases, which is consistent with our experimental results (Fig. 4).

The deformation modes of the hierarchical nanostructured Al-based alloy and nanocrystalline $\mathrm{Al}$ were further compared. 'G1' in Fig. $1 \mathrm{~b}$ is selected and compared with the same grain in polycrystalline $\mathrm{Al}$, at a strain of 7.5\% (Supplementary Fig. 9). The length scale of grain along the compression direction changes from 10 to $8 \mathrm{~nm}$ (Supplementary Fig. 9b) in the hierarchical nanostructured Al-based alloy while it remains $10 \mathrm{~nm}$ in polycrystalline $\mathrm{Al}$ (Supplementary Fig. 9e) at a strain of 7.5\%. In addition, similar dislocation propagation trajectories are observed in both of them (Supplementary Fig. $9 \mathrm{c}$ and $\mathrm{f}$ ). It indicates that the interaction between dislocations and MGs are different from the traditional dislocation-grain boundary (GB) interaction, which account for the great ductility of our hierarchical nanostructured Al-based alloy. It should be emphasized that the length scale of grains gradually decreases along the compression direction, which could be an important factor for strengthening during plastic deformation since it is equivalent to grain refinement ${ }^{55}$.

In fact, the initiation of first dislocation, which represents the start of plastic deformation, occurs at a strain of $\sim 5.5 \%$ and $\sim 3.5 \%$ for the hierarchical nanostructured $\mathrm{Al}$-based alloy and polycrystalline $\mathrm{Al}$, respectively. That means the hierarchical nanostructured Al-based alloy can endure larger elastic stage, i.e., the initiation of dislocations from the glass/crystal interface is more difficult than polycrystalline $\mathrm{Al}$, which could be evidence for the better mechanical stability of glass/crystal interface than GBs. In order to clearly identify the relationship between GBs and glass/crystal interface, a layered model was built (Supplementary Fig. 10a) and the deformation behavior under compression was simulated. Supplementary Fig. 10b-e show the successive snapshots of the initiation and propagation of dislocations from GBs. However, as a comparison, no dislocation initiates from glass/crystal interface, which indicates that the glass/crystal interface has better efficiency to impede dislocation motion than GB.

The interaction between dislocations and the MG phase was studied. The GB was replaced by $\mathrm{Al}_{85} \mathrm{Ni}_{15} \mathrm{MG}$ in Supplementary Fig. 11a. It can be determined that the dislocations mainly nucleate from the glass/crystal interface, which has also been demonstrated in Supplementary Movie 1. Convex hulls (red circles in Supplementary Fig. 11b, c) appear on the glass/crystal interface, which results from the interaction between dislocations and the MG phase. The atomic shear strain caused by dislocations motion and MG flow can clearly be characterized in Supplementary Fig. 11e, f. More importantly, the regions pointed by orange arrows in Supplementary Fig. 11f are the MG regions that exhibit relatively large atomic shear strain. They are close to the positions where dislocation-interface interaction occurs. In contrast, larger atomic shear strain is not observed for the MG atoms close to the glass/crystal interface (the red dashed-rectangle region in Supplementary Fig. 11f), where no dislocation nucleation or accommodation occurs. In addition, Supplementary Fig. 12 directly shows that the roughness of the glass/crystal interface greatly increases after plastic deformation, which identifies the structural synergy during plastic deformation. Thus, these large atomic shear strain regions are mainly activated by the dislocation nucleation or

accommodation, and the MG atoms can locally reconcile the atomic structure to endure large plastic deformation.

At last, the variation of crystal fraction was calculated after the compression in simulation (Supplementary Fig. 13). No matter compressing the sample along $x$ - or $y$-direction, the fraction of crystal almost remains unchanged. This proves that no mechanical instability such as amorphization of $\mathrm{Al}$ grains and crystallization of $\mathrm{Al}_{85} \mathrm{Ni}_{15}$ MGs occurs, even at a large strain of $30 \%$.

A hierarchical nanostructured Al-based alloy is studied by MD simulation and an extraordinary grain-flattening based plastic deformation behavior is successfully predicted. On one hand, dislocations nucleate more difficultly from the glass/ crystal interface compared with the GB, which contribute to ultrahigh strength. On the other hand, a series of detailed MD studies help us to understand the origin of the exceptional properties of this hierarchical nanostructured Al-based alloy. The shear deformation is constrained at the glass/crystal interface and cannot cross the MG interior. Furthermore, the dislocation-based mechanism dominates the plastic deformation of the crystalline phase, which contributes to the large plasticity. The strong MG phase in the hierarchical nanostructured Al-based alloy impedes the large shear failure and well coordinate the plasticity by plastic flow.

\section{Data availability}

The data that support the findings of this study are available from the corresponding author upon reasonable request.

Received: 6 March 2019; Accepted: 18 October 2019;

Published online: 08 November 2019

\section{References}

1. Callister Jr, W. Materials Science and Engineering: An Introduction (Wiley 1994).
2. George, E. P., Raabe, D. \& Ritchie, R. O. High-entropy alloys. Nat. Rev. Mater. 4, 515-534 (2019)

3. Lu, K., Lu, L. \& Suresh, S. Strengthening materials by engineering coherent internal boundaries at the nanoscale. science 324, 349-352 (2009).

4. Li, Z., Tasan, C. C., Pradeep, K. G. \& Raabe, D. A TRIP-assisted dual-phase high-entropy alloy: grain size and phase fraction effects on deformation behavior. Acta Mater. 131, 323-335 (2017).

5. Greer, A. L. Metallic glasses. Science 267, 1947-1953 (1995).

6. Schuh, C. A. \& Lund, A. C. Atomistic basis for the plastic yield criterion of metallic glass. Nat. Mater. 2, 449-452 (2003).

7. Inoue, A., Shen, B., Koshiba, H., Kato, H. \& Yavari, A. R. Cobalt-based bulk glassy alloy with ultrahigh strength and soft magnetic properties. Nat. Mater. 2, 661-663 (2003).

8. Amiya, K., Urata, A., Nishiyama, N. \& Inoue, A. Fe-B-Si-Nb bulk metallic glasses with high strength above $4000 \mathrm{MPa}$ and distinct plastic elongation. Mater. Trans. 45, 1214-1218 (2004)

9. Zhang, Y. \& Greer, A. Thickness of shear bands in metallic glasses. Appl. Phys. Lett. 89, 071907 (2006)

10. Ketov, S. et al. Rejuvenation of metallic glasses by non-affine thermal strain Nature 524, 200-203 (2015)

11. Hofmann, D. C. et al. Designing metallic glass matrix composites with high toughness and tensile ductility. Nature 451, 1085-1089 (2008).

12. Pauly, S., Gorantla, S., Wang, G., Kühn, U. \& Eckert, J. Transformationmediated ductility in CuZr-based bulk metallic glasses. Nat. Mater. 9, 473-477 (2010).

13. Wu, Y., Xiao, Y., Chen, G., Liu, C. T. \& Lu, Z. Bulk metallic glass composites with transformation-mediated work-hardening and ductility. Adv. Mater. 22, 2770-2773 (2010).

14. Zhang, J., Liu, G., Lei, S., Niu, J. \& Sun, J. Transition from homogeneous-like to shear-band deformation in nanolayered crystalline $\mathrm{Cu}$ /amorphous $\mathrm{Cu}-\mathrm{Zr}$ micropillars: Intrinsic vs. extrinsic size effect. Acta Mater. 60, 7183-7196 (2012).

15. Kim, J. Y. et al. Suppression of catastrophic failure in metallic glass-polyisoprene nanolaminate containing nanopillars. Adv. Funct. Mater. 22, 1972-1980 (2012).

16. Guo, W. et al. Intrinsic and extrinsic size effects in the deformation of amorphous CuZr/nanocrystalline Cu nanolaminates. Acta Mater. 80, 94-106 (2014).

17. Wang, Y., Li, J., Hamza, A. V. \& Barbee, T. W. Ductile crystalline-amorphous nanolaminates. Proc. Natl Acad. Sci. USA 104, 11155-11160 (2007).

18. Jang, D. \& Greer, J. R. Transition from a strong-yet-brittle to a stronger-andductile state by size reduction of metallic glasses. Nat. Mater. 9, 215-219 (2010).

19. Guo, H. et al. Tensile ductility and necking of metallic glass. Nat. Mater. 6, 735-739 (2007).

20. Tian, L. et al. Approaching the ideal elastic limit of metallic glasses. Nat Commun. 3, 609 (2012).

21. Chen, M. et al. Deformation twinning in nanocrystalline aluminum. Science 300, 1275-1277 (2003).

22. Wu, G., Chan, K.-C., Zhu, L., Sun, L. \& Lu, J. Dual-phase nanostructuring as a route to high-strength magnesium alloys. Nature 545, 80-83 (2017).

23. Inoue, A., Ohtera, K., Tsai, A.-P., Kimura, H. \& Masumoto, T. Glass transition behavior of Al-Y-Ni and Al-Ce-Ni amorphous alloys. Jpn. J. Appl. Phys. 27, L1579 (1988).

24. Zhang, Z. et al. Glass forming ability and crystallization behavior of Al-Ni-RE metallic glasses. Intermetallics 42, 23-31 (2013).

25. Miracle, D. B. A structural model for metallic glasses. Nat. Mater. 3, 697-702 (2004).

26. Hirata, A. et al. Direct observation of local atomic order in a metallic glass. Nat. Mater. 10, 28-33 (2011).

27. Cantwell, P. R. et al. Grain boundary complexions. Acta Mater. 62, 1-48 (2014).

28. Khalajhedayati, A., Pan, Z. \& Rupert, T. J. Manipulating the interfacial structure of nanomaterials to achieve a unique combination of strength and ductility. Nat. Commun. 7, 10802 (2016).

29. Shi, X. \& Luo, J. Grain boundary wetting and prewetting in Ni-doped Mo. Appl. Phys. Lett. 94, 251908 (2009).

30. Li, Q. et al. High-Strength Nanotwinned Al Alloys with 9R Phase. Adv. Mater 30, 1704629 (2018)

31. Louzguine, D. \& Inoue, A. Crystallization behaviour of Al-based metallic glasses below and above the glass-transition temperature. J. Non-cryst. Solids 311, 281-293 (2002).

32. Yu, C., Sun, P., Kao, P. \& Chang, C. Mechanical properties of submicrongrained aluminum. Scr. Mater. 52, 359-363 (2005).

33. Shim, S., Bei, H., George, E. P. \& Pharr, G. M. A different type of indentation size effect. Scr. Mater. 59, 1095-1098 (2008). 
34. Uchic, M. D., Dimiduk, D. M., Florando, J. N. \& Nix, W. D. Sample dimensions influence strength and crystal plasticity. Science 305, 986-989 (2004).

35. Wang, L. et al. Grain rotation mediated by grain boundary dislocations in nanocrystalline platinum. Nat. Commun. 5, 4402 (2014).

36. Wang, L. et al. New twinning route in face-centered cubic nanocrystalline metals. Nat. Commun. 8, 2142 (2017).

37. Van Leer, B., Genc, A. \& Passey, R. Ga+ and Xe+ FIB Milling and Measurement of FIB Damage in Aluminum. Microsc. Microanal. 23, 296-297 (2017).

38. Xiao, Y. et al. Investigation of the deformation behavior of aluminum micropillars produced by focused ion beam machining using $\mathrm{Ga}$ and Xe ions. Scr. Mater. 127, 191-194 (2017).

39. Lei, Z. et al. Enhanced strength and ductility in a high-entropy alloy via ordered oxygen complexes. Nature 563, 546-550 (2018).

40. Li, Z., Pradeep, K. G., Deng, Y., Raabe, D. \& Tasan, C. C. Metastable highentropy dual-phase alloys overcome the strength-ductility trade-off. Nature 534, 227-230 (2016).

41. Liu, Y. H., Wang, G., Wang, R. J., Pan, M. X. \& Wang, W. H. Super plastic bulk metallic glasses at room temperature. Science 315, 1385-1388 (2007).

42. Fang, T., Li, W., Tao, N. \& Lu, K. Revealing extraordinary intrinsic tensile plasticity in gradient nano-grained copper. Science 331, 1587-1590 (2011).

43. Lu, L., Sui, M. \& Lu, K. Superplastic extensibility of nanocrystalline copper at room temperature. Science 287, 1463-1466 (2000).

44. $\mathrm{Xu}, \mathrm{W}$., Liu, X. \& Lu, K. Strain-induced microstructure refinement in pure $\mathrm{Al}$ below $100 \mathrm{~nm}$ in size. Acta Mater. 152, 138-147 (2018).

45. Schiøtz, J., Di Tolla, F. D. \& Jacobsen, K. W. Softening of nanocrystalline metals at very small grain sizes. Nature 391, 561-563 (1998).

46. Wat, A. et al. Bioinspired nacre-like alumina with a bulk-metallic glassforming alloy as a compliant phase. Nat. Commun. 10, 961 (2019).

47. Plimpton, S. Fast parallel algorithms for short-range molecular dynamics. J. Comput. Phys. 117, 1-19 (1995).

48. Pun, G. P. P. \& Mishin, Y. Development of an interatomic potential for the Ni-Al system. Philos. Mag. 89, 3245-3267 (2009).

49. Zhu, J. B., Wang, S., Qiao, M. H., Wang, W. N. \& Fan, K. N. First-principle molecular dynamics study of the structural and electronic properties of liquid and amorphous Ni-Al alloys. J. Non-Cryst. Solids 353, 2638-2645 (2007).

50. Yu, C. Y. et al. Ab initio molecular dynamics simulation of the atom packing and density of Al-Ni amorphous alloys. Sci. China-Technol. Sci. 53, 3175-3182 (2010).

51. Shimizu, F., Ogata, S. \& Li, J. Theory of shear banding in metallic glasses and molecular dynamics calculations. Mater. Trans. 48, 2923-2927 (2007).

52. Clarke, A. S. \& Jonsson, H. Structural changes accompanying densification of random hard-sphere packings. Phys. Rev. E 47, 3975-3984 (1993).

53. Cheng, Z., Zhou, H., Lu, Q., Gao, H. \& Lu, L. Extra strengthening and work hardening in gradient nanotwinned metals. Science 362, eaau1925 (2018)

54. Pan, Q., Zhou, H., Lu, Q., Gao, H. \& Lu, L. History-independent cyclic response of nanotwinned metals. Nature 551, 214-217 (2017).

55. Liu, X. C., Zhang, H. W. \& Lu, K. Strain-induced ultrahard and ultrastable nanolaminated structure in nickel. Science 342, 337-340 (2013).

\section{Acknowledgements}

J. Lu gratefully acknowledges that the work was partially supported by the National Key R\&D Program of China (Project No. 2017YFA0204403), the Major Program of the
National Natural Science Foundation of China (NSFC) grant 51590892, the Hong Kong Collaborative Research Fund (CRF) Scheme (Ref. C4028-14G, C4026-17W), Themebased Research Scheme (Ref. T13-402/17-N), General Research Fund (GRF) Scheme (CityU 11247516, CityU 11209918 and CityU 11216219). B.A. Sun thanks the support from NSFC with grant No. 51822107. Atom probe tomography research was conducted at the Inter-University 3D Atom Probe Tomography Unit of City University of Hong Kong, which is supported by the CityU grant 9360161 and CRF grant C1027-14E. We would like to thank Z.F. Zhou for magnetron sputtering assistance at City University of Hong Kong.

\section{Author contributions}

J. Lu designed the project; G. Wu, B.A. Sun, and J. Lu designed the material; G. Wu and C. Liu designed the experiments; G. Wu conducted nanoindentation, SEM and TEM in situ tension experiments; C. Liu conducted SEM in situ micro-compression, TEM characterization, and FIB experiments; B. Han, J.H. Luan, J.J. Kai, and C.T. Liu conducted APT characterization; K. Cao, G. Wu, and Y. Lu conducted TEM in situ compression experiment; L.Z. Cheng provided the shellfish sample; G. Wu, C. Liu, Q. Wang, and B.A. Sun analyzed the data; L.G. Sun performed the MD simulation and wrote the corresponding text. $\mathrm{Ge} \mathrm{Wu}, \mathrm{C}$. Liu, and J. Lu wrote the main manuscript; all authors contributed to the discussion of the results.

\section{Competing interests}

The authors declare no competing interests.

\section{Additional information}

Supplementary information is available for this paper at https://doi.org/10.1038/s41467 019-13087-4.

Correspondence and requests for materials should be addressed to J.L.

Peer review information Nature Communications thanks Matthew Kramer, Simon Pauly and the other, anonymous, reviewer for their contribution to the peer review of this work.

Reprints and permission information is available at http://www.nature.com/reprints

Publisher's note Springer Nature remains neutral with regard to jurisdictional claims in published maps and institutional affiliations.

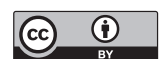

Open Access This article is licensed under a Creative Commons Attribution 4.0 International License, which permits use, sharing, adaptation, distribution and reproduction in any medium or format, as long as you give appropriate credit to the original author(s) and the source, provide a link to the Creative Commons license, and indicate if changes were made. The images or other third party material in this article are included in the article's Creative Commons license, unless indicated otherwise in a credit line to the material. If material is not included in the article's Creative Commons license and your intended use is not permitted by statutory regulation or exceeds the permitted use, you will need to obtain permission directly from the copyright holder. To view a copy of this license, visit http://creativecommons.org/ licenses/by/4.0/.

(C) The Author(s) 2019 